BULL. AUSTRAL. MATH. SOC.

\title{
AUTOMORPHISM GROUPS OF ORTHOMODULAR LATTICES
}

\author{
GUDRUN KALMBACH
}

Every group is the automorphism group of an orthomodular lattice.

In this note we prove that every group is the automorphism group of some orthomodular lattice. The proof makes use of the following results: Birkhoff's construction of a lattice with a given group as its automorphism group [4]; the embedding of a lattice $L$ in an orthomodular lattice generated by the chains in $L$ [6]; the existence of Boolean algebras with trivial automorphism groups [7] and the pasting of orthomodular lattices at atoms [5]. The case of finite groups was solved in [11], it is an easy application of results in [10]. For basic facts about orthomodular lattices the reader is referred to [5].

Let $G$ be a group provided with a ordinal-type well ordering < such that the unit $e \in G$ is the smallest element. The lattice $L$ consists of the elements of the following disjoint sets: $\{0\},\{1\}, G, G \times G$ and the set $U$ of two-element subsets $\{x, y\}$ of $G$. The order structure $\leq$ on $L$ has 0 as the smallest, $I$ as the largest element and $G$ as its set of atoms. Furthermore we define

$$
\begin{aligned}
& \text { (i) } x, y<\{x, y\} \in U \text { for } x, y \in G, \\
& \text { (ii) }(x, y) \leq(u, v) \text { if } x=u \text { and } y \leq v \text { for } \\
& (x, y),(u, v) \in G \times G, \\
& \text { (iii) } x \leq(y, z) \text { if } x^{-1} y \leq z \text { for } x \in G,(y, z) \in G \times G,
\end{aligned}
$$

Received 28 November 1983.

Copyright Clearance Centre, Inc. Serial-fee code: 0004-9727/84 $\$ A 2.00+0.00$. 


$$
\begin{aligned}
& \text { (iv) }\{x, y\} \leq(x, z) \text { for }\{x, y\} \in U,(x, z) \in G \times G \text { if } \\
& y \leq(x, z) .
\end{aligned}
$$

The maximal chains in $L$ are of the form

$$
0<x<(x, e)<\ldots<(x, a)<\ldots<1
$$

or

$$
0<x<\{x, y\}<\left(x, b_{0}\right)<\ldots<(x, a)<\ldots<1
$$

or

$$
0<x<\{x, y\}<\left(y, c_{0}\right)<\ldots<(y, d)<\ldots<1
$$

where $b_{0}$ respectively $c_{0}$ are the smallest elements in $G$ with $y^{-1} x \leq b_{0}$ respectively $x^{-1} y \leq c_{0}$. Observe that the only non-trivial joins and meets in $L$ are $x \vee y=\{x, y\}=\left(x, b_{0}\right) \wedge\left(y, c_{0}\right)$.

The automorphisms $g$ of $L$ are given by

$$
\begin{aligned}
g(0) & =0, g(1)=1, g(x)=b x, \\
g(x, y) & =(b x, y), g(\{x, y\})=\{b x, b y\}
\end{aligned}
$$

for a fixed element $b \in G$ ([4], pp. 44-47). Therefore $G$ is isomorphic to the automorphism group of $L$.

The lattice $L$ generates an orthomodular lattice $M$ as follows (for details see [6]): let $\left(C_{i}\right)_{i \in I}$ by the set of finite chains in $L$ containing 0,1 and define $D_{i j}=C_{i} \cap C_{j}$. Every $C_{i}$ generates a Boolean algebra $B_{i}$. In the union $M=\underset{i \in I}{U} B_{i}$ the elements of $B_{i}$ and $B_{j}$ which are generated by the common subchain $D_{i j}$ are identified. $M$ is an orthomodular lattice with the ' and $\leq$ structure induced by the $B_{i}$. The atoms of $M$ are the elements of $G$, of $H=\left\{\{x, y\} \wedge x^{\prime} \mid x, y \in G\right\}$, of $P=\left\{\left(x, b_{0}\right\} \wedge\{x, y\}^{\prime} \mid\{x, y\} \in U, b_{0}\right.$ minimal with $\left.y^{-1} x \leq b_{0}\right\}$ and of $Q=\left\{(x, c) \wedge(x, a)^{\prime} \mid x \in G, c\right.$ covers $\left.a\right\}$.

An automorphism of $M$ maps atoms onto atoms. Therefore we now extend $M$ to an orthomodular lattice $N$ with the same set of atoms by pasting 
suitable automorphism-free Boolean algebras at the atoms of $M-L$ in such a manner that $N$ has the (uniquely) extended automorphisms of $L$ as its automorphisms. Let $B_{\tau}$ be a Boolean algebra of power $2^{X_{\tau}}$ ( $\tau$ an ordinal) with $\operatorname{Aut}\left(B_{\tau}\right)=1$ [7]. Then $B_{\tau}$ has at most one atom. We can assume that it has exactly one atom since if it has no atom then $2 \times B_{\tau}$ has one atom and $\operatorname{Aut}\left(2 \times B_{\tau}\right)=1$.

LEMMA. If $a \in C$ is an atom of the orthomodular lattice $C$ and $D$ is obtained from the disjoint union of $C$ and $B_{\tau}$ by identifying the two zero elements, the two wnit elements, the atom $a \in C$ with the atom $b \in B_{\tau}$ and the element $a^{\prime} \in C$ with $b^{\prime} \in B_{\tau}$ then $D$ with the ' and $\leq$ structure induced by $C$ and $B_{\tau}$ is an orthomodular lattice.

This is a special case of Greechie's paste job ([5], pp. 42-44). We assume now that $|G|$ is infinite. Then all blocks (maximal Boolean subalgebras) of $M$ have the same cardinality $m$. Choose the ordinal number $\eta$ such that $2^{X} \eta>m$. To every atom in $H$ respectively $P$ we paste one copy of $B_{\eta}$ respectively $B_{\eta+1}$. Enumerate the elements of the well-ordered chain $G$ by

$$
e=a_{n+2}<a_{n+3}<\ldots<a_{\sigma}<\ldots .
$$

At every atom $\left(x, a_{\sigma}\right) \wedge\left(x, a_{\sigma-1}\right)^{\prime} \in Q$ (if it exists for $\sigma$ ) we paste one copy of $B_{\sigma}$. The lemma assures that the pasting $N$ of $M$ with all these Boolean algebras is an orthomodular lattice.

If an atom $p$ of $N$ is mapped by an automorphism of $N$ to an atom $q \in N$ then the blocks containing $p$ are mapped onto the blocks containing $q$. For different $\sigma_{1}, \sigma_{2}, B_{\sigma_{1}}$ is not isomorphic to $B_{\sigma_{2}}$ and $B_{\sigma_{1}}$ is, for $\sigma_{1} \geq n$, also not isomorphic to some block in $M$ by cardinality reasons. Hence an automorphism of $N$ can only map atoms of $L$ onto atoms of $L$, atoms of $H$ respectively $P$ onto atoms of $H$ respectively $P$ and in $Q$ only the atoms of the form $\left(x, a_{\sigma}\right) \wedge\left(x, a_{\sigma-1}\right)^{\prime}$ and $\left(y, a_{\sigma}\right) \wedge\left(y, a_{\sigma-1}\right)^{\prime}$ can be mapped onto one another. Every automorphism 
of $L$ therefore extends uniquely to an automorphism of $N$ and $N$ has no other automorphisms which shows that $\operatorname{Aut}(N)$ is isomorphic to $G$.

\section{References}

[1] G. Birkhoff, Lattice theory, 3rd edition (American Mathematical Society Colloquium Publications, 25. American Mathematical Society, Providence, Rhode Island, 1973).

[2] R. Greechie, "Finite groups as automorphism groups of orthocomplemented projective planes", J. Austral. Math. Soc. 25 (1978), 19-24.

[3] S. Gudder, "Representations of groups as automorphisms on orthomodular lattices and posets", Canad. J. Math. 23 (1971), 659-673.

[4] B. Jónsson, Topics in universal algebra (Lecture Notes in Mathematics, 250. Springer-Verlag, Berlin, Heidelberg, New York, 1972).

[5] G. Kalmbach, Orthomodular Zattices (Academic Press, London, 1983).

[6] G. Kalmbach, "Orthomodular lattices do not satisfy any special lattice equation", Arch. Math. (Basel) 28 (1977), 7-8.

[7] R. McKenzie and J. Monk, "On automorphism groups of Boolean algebras", Infinite and finite sets, 951-988 (Colloq. Math. Soc. J. Bolyai, 10. North-Holland, Amsterdam, 1973).

[8] R. Morash, "Angle bisection and orthoautomorphisms in Hilbert lattices", Conad. J. Math. 25 (1973), 261-272.

[9] J. Rosenstein, Linear orderings (Academic Press, New York, 1982).

[10] G. Sabidussi, "Graphs with given group and given graph-theoretical properties", Conad. J. Math. 9 (1957), 515-525. 
[11] G. Schrag, "Every finite group is the automorphism group of some finite orthomodular lattice", Proc. Amer. Math. Soc. 55 (1976), 243-249.

Abt. Math. III. O.E.,

Universitat UIm,

D-7900 UIm,

Federal Republic of Germany

and

Department of Mathematics, Institute of Advanced Studies, Australian National University, GPO Box 4, Canberra, ACT 2601, Australia. 